OPEN

SUBJECT AREAS:

MOLECULAR SELF-

ASSEMBLY

NANOPARTICLES

Received

25 November 2013

Accepted

7 January 2014

Published

27 January 2014

Correspondence and requests for materials should be addressed to

H.L. (lihuilmy@163.

com)

\title{
How Does Carbon Nanoring Deform to Spiral Induced by Carbon Nanotube?
}

\author{
Wei Chen \& Hui Li
}

Key Laboratory for Liquid-Solid Structural Evolution and Processing of Materials, Ministry of Education, Shandong University, Jinan 250061, People's Republic of China.

Molecular dynamics (MD) simulations have been performed on the interaction between carbon nanoring (CNR) and single-wall carbon nanotube (SWCNT). The results show that, the CNR can spontaneously insert into the hollow interior of the SWCNTs to form a DNA-like double-helix, or collapse to a linked double graphitic nanoribbon and wrap in a helical manner around a tube. Further analyses of energy components show that this unique phenomenon is the result of the Van der Waals interaction. The spiral configuration of the CNR takes the least amount of energy and achieves the maximum occupancy. The sizes of CNR and SWCNT should meet the required conditions to guarantee the spiral form in the insertion and wrapping processes. Two CNRs can also be encapsulated in the SWCNT to form a helix at the same time. Furthermore, we also studied the encapsulation process of $\mathrm{CNRs}$ modified with $-\mathrm{OH}$ and $-\mathrm{H}$ functional groups.

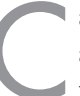

arbon nanotubes (CNTs), due to their unique structure and distinctive properties, have attracted intense attention on both theoretical researches and potential applications in many fields ${ }^{1}$. Due to their conventional hollow interior ${ }^{2}$ and large specific surface area, single-walled carbon nanotubes (SWCNTs) can serve as a nanometer-sized mold or template, which has aroused great interest in the research field of CNTsbased composite nanostructure fabrication. The composites integrated with SWCNTs and other materials have considerable influence on their toughness, crystalline morphology ${ }^{4}$, mechanical strength, etc. Previous theoretical and experimental results have shown that open-ended nanotubes could act as "molecular straws" capable of absorbing dipolar molecules through capillary action ${ }^{5}$. The presence of encapsulated "foreign" nanostructures in SWCNTs is known to tremendously affect the properties of the pristine tube and the fillers, giving rise to unique nanostructures with exciting new applications. The encapsulation of a series of materials within SWCNTs, including metals ${ }^{6,7}$, water ${ }^{8,9}$, fulleren $e^{10,11}$, and grapheme ${ }^{12,13}$, as well as the properties of the fillers have been discussed for the functionalization of CNTs. At the same time, polymer chains such as DNA can wrap in a helical manner around a CNT with periodic pitch ${ }^{14,15}$. This non-covalent "wrapping" phenomenon can also be utilized to drive self-assembly ${ }^{16,17}$, and alter the functionalization of the tubes ${ }^{18}$. On one hand, the fillers in the confined space possess novel properties which are quite different from those of their bulk counterparts. On the other hand, the carbon shell can be regarded as a natural layer of fillers protecting against oxidation and shape fragmentation ${ }^{19}$. Therefore, the "CNTs-based composite" has great potential in hydrogen storage, heterogeneous catalysis ${ }^{20}$, nanodevices, magnetic data storage, electromagnetic wave absorption, as well as drug and gene delivery in the field of biology ${ }^{21,22}$.

Although there have been large quantities of important discoveries regarding the insertion and wrapping of various materials, studies concerning the encapsulation of carbon nanoring (CNR) into the SWCNTs and the wrapping of the CNR outside the wall of the SWCNTs have yet to be performed. The super-short carbon nanorings (CNRs) can also be considered as rolled and enclosed narrow graphene nanoribbons (GNRs) ${ }^{23}$. CNRs with large diameters are susceptible to deform their cylindrical symmetry to various structural morphologies $^{24}$, such as oval, peanut, or even ribbon shape. Moreover, the radical deformation of the CNR can affect its mechanical ${ }^{25}$ and electrical properties ${ }^{26}$. The self-deformation and collapse $\mathrm{e}^{27}$ of the CNRs make themselves insert into SWCNTs and wrap around the SWCNTs possibly. It is always very difficult to control the length of CNRs during the synthesis process ${ }^{28}$. Recently, Sun et al. took advantage of the confined space of the two-dimensional interlayer galleries of a layered double hydroxide (LDH) host to limit the in situ growth of CNRs, controlling the length down to the molecular scale of $\sim 1 \mathrm{~nm}^{23}$. Therefore, a convincing model by which we may thoroughly understand the interaction between the CNRs and SWCNTs becomes very valuable. In the present study, systematic theoretical investigations are performed to demonstrate how the CNR interacts with the SWCNT. In addition, the possible interacting mechanism is examined to establish the nature of the self-assembly of CNRs 


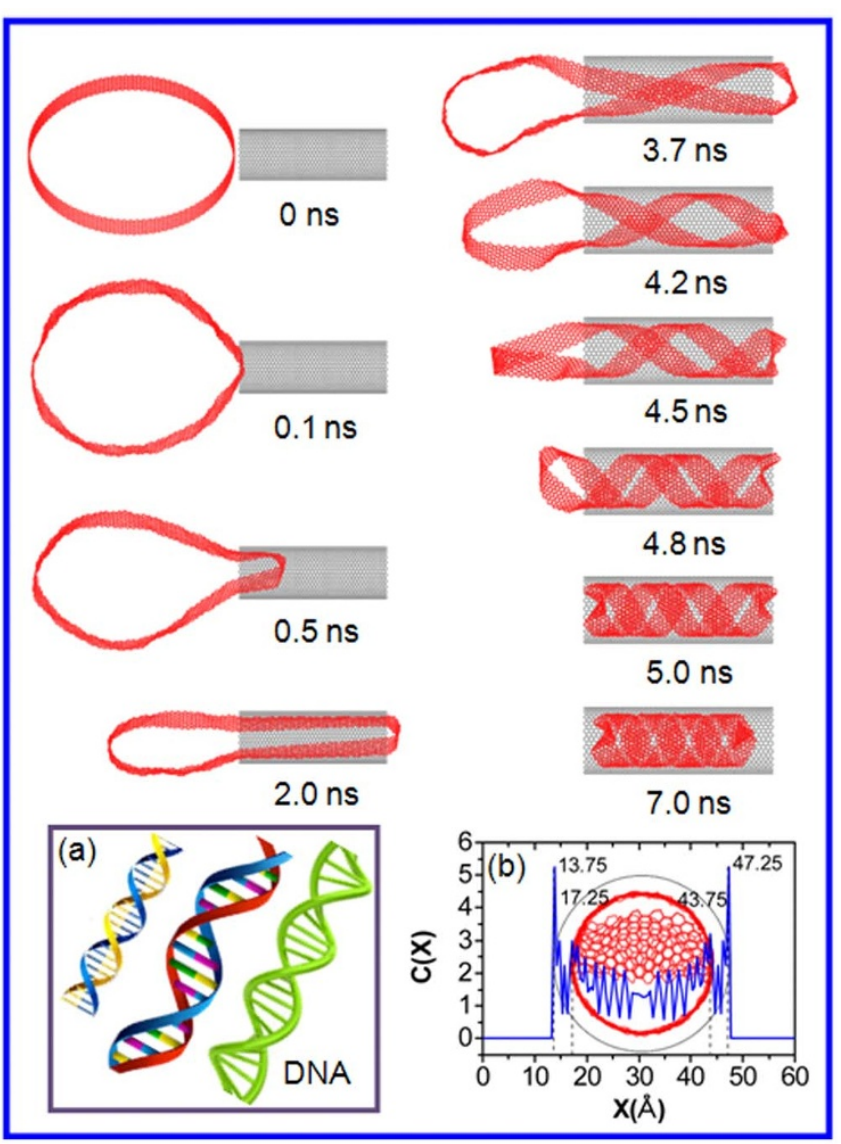

Figure $1 \mid$ Representative snapshots of a CNR $(100,100)$ inserting into the SWCNT $(25,25)$ to form a helical configuration. The length of the CNR is $14.76 \AA$ A. (a) Sketch of DNA chains which has a double spiral configuration. (b) Concentration distribution profiles of the CNR and SWCNT in the systems in the X-direction.

and SWCNTs. This study is not only helpful for more thoroughly understanding the properties of CNRs at an atomistic level, but also is essential to the substantial potential application for fabricating functional nanodevices.

\section{Results}

Figure 1 provides the representative snapshots of the CNR $(100,100)$ with the length of $14.76 \AA$, inserted helically into the SWCNT (25, 25). First, the CNR is placed at the center of the SWCNT entrances separated by $5 \AA$, with the axes of the CNR and SWCNT perpendicular to each other. When the simulation begins, the CNR with a diameter of $135.6 \AA$ tends to be thermodynamically unstable and its cylindrical symmetry is collapsed. Owing to the Van der Waals interaction ${ }^{29}$ between the CNR and SWCNT, the CNR stretches its cross-section and approaches the SWCNT at $0.1 \mathrm{~ns}$. As the simulations progress, the portion of the CNR near the entrance is captured by the inner hollow space of the SWCNT and gradually moves forward along the inner wall of the SWCNT, in which the CNR are held tightly against SWCNT due to the interaction between them. After an initial correlation time, the CNR begins to curl in the hollow interior of the SWCNT (at $\mathrm{t}=3.5 \mathrm{~ns}$ ). When the simulation time reaches $4.8 \mathrm{~ns}$, the CNR displays a clear spiral conformation with a large helical pitch, attempting to occupy the entirety of the tubes. Then the spirals become denser due to the Van der Waals interaction. Eventually, a perfect DNA-like double-helix (Figure 1(a)), with remarkably constant pitches between neighboring spirals forms in the SWCNT. In addition, we further simulate the influence of the temperature on the final structure of the CNR-SWCNT system. It is surprising that the final composite structure of the CNR-SWCNT system can maintain stability even when the temperature is $1000 \mathrm{~K}$.

This final geometric configuration of the CNR-SWCNT system can be further characterized by the concentration distribution profile between the CNR and SWCNT in the X-direction. The separation of the adjacent layers can be obtained by the distance between two neighboring peaks in the concentration profiles. From the peak details labeled in Figure 1(b), the separation between the inner layer of CNR and the SWCNT is about $3.5 \AA$, which is very close to the wall thickness of the multiwalled CNTs ( $3.4 \AA$ ).

We further study the insertion processes of CNRs with the same diameter, namely $135.60 \AA$, but different lengths, i.e. 7.38, 9.84, $12.30,14.76,17.22 \AA$. Figure 2 (a) shows the diameter threshold of SWCNT successfully encapsulated by the CNR with certain lengths. We observe that the diameter of the SWCNT should be larger than the threshold to ensure the collapse and insertion of the CNR with certain lengths. If the diameter of the nanotube is too small, the Van der Waals interaction cannot exceed the energy barrier, and the shell distance between carbon atoms cannot maintain $3.5 \AA$ due to the

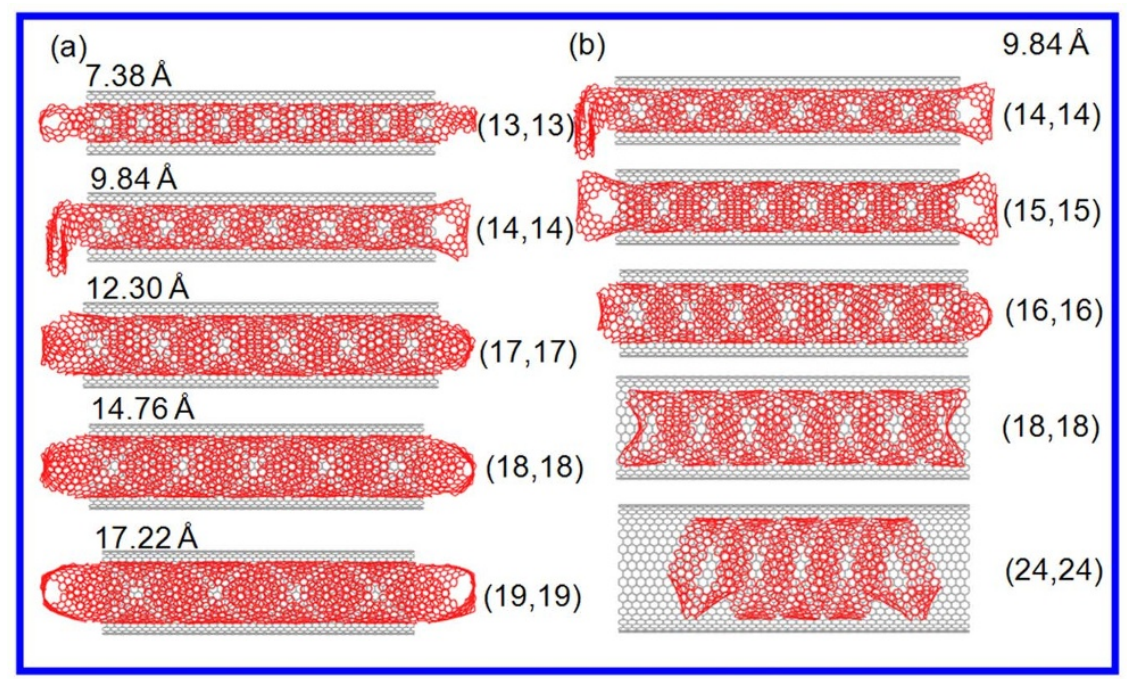

Figure $2 \mid$ (a) The critical diameters of the SWCNTs to guarantee the insertion of the CNR $(100,100)$ successfully: CNR(7.38 $\AA$ )@SWCNT(13,13), CNR(9.84 ̊)@SWCNT(14,14), CNR(12.30 ̊)@SWCNT(17,17), CNR(14.76 ̊)@SWCNT(18,18), CNR(17.22 ̊)@SWCNT(19,19), respectively. (b) The helical insertion of CNR $(100,100)$ with length of $9.84 \AA$ into the SWCNT with different diameters. 
confinement. Consequently, the sizes of SWCNTs should be chosen properly according to the lengths of the CNRs to facilitate the insertion. In order to verify the final configurations of the $\operatorname{CNR}(100,100)$ with a length of $9.84 \AA$ in the varied SWCNTs, a series of armchair SWCNTs $(n, n)$ are selected with the indices of $\mathrm{n}=14,15,16,18$ and 24 , yielding the tube diameters of $18.98,20.34,21.70,24.41$ and $32.54 \AA$, respectively, and the tube length is set to $98.38 \AA$ to fit the CNR. Figure 2(b) demonstrates that a perfect helix inside the nanotube can be obtained after the CNRs are trapped by the inner wall of the tube, which is independent of the size of the tube.

What would happen if the length of the SWCNT is too short? Figure 3 shows the overall insertion process of the CNR $(100,100)$ with the length of $14.76 \AA$ into the SWCNT $(25,25)$. The length of the SWCNT is chosen as $24.60 \AA$. It is worth noting that the CNR can insert into the SWCNT successfully with the trapped portion to form a helix. When the simulation proceeds, the front helical structure is pushed out and the portion outside the tube continuously enters the SWCNT in a helical manner. Finally, the CNR attempts to be symmetric to the tube, with the middle portion of the CNR in the inner wall of the tube forming a perfect helical configuration.

In the above discoveries, we have mainly focused on the insertion of one CNR into the SWCNT. Next, we will clarify how two CNRs insert into the SWCNT. As shown in Figure 4, the two CNRs with the same diameter and length are placed at the two ends of the SWCNT, with their axes perpendicular to each other. When the simulation begins, the two CNRs are captured by the inner hollow of the SWCNT and move forward along the wall of the tube, due to the Van der Waals interaction. When they contact with each other, one of them is pushed down to a graphitic nanoribbon, as a result of the competition between the two CNRs. When the simulation time reaches $2.25 \mathrm{~ns}$, helices arise to both of the CNRs. When the simulation time $\mathrm{t}=5.0 \mathrm{~ns}$, the CNRs display a clear helical conformation. The decreased total potential energy $\mathrm{E}_{\mathrm{P}}$ and negative Van der Waals interaction energy $\Delta \mathrm{E}_{\mathrm{vdW}}$, which are synchronous to each other, indicate that the self-assembly course is spontaneous and the adhesion is strong.

The unique spontaneous encapsulation of CNRs into SWCNTs arouses our interest to investigate the delivery of the materials, as this can be utilized to deliver substances into the nanoscale confined space without any other external force. In order to explore the possible applications, we simulate the interaction between the SWCNTs and CNRs modified with different functional groups. We select the OH modified CNR $(100,100)$ with the length of $14.76 \AA$ encapsulated into the SWCNT $(30,30)$. As shown in Figure 5, the CNR modified with the $-\mathrm{OH}$ can spontaneously be inserted into the

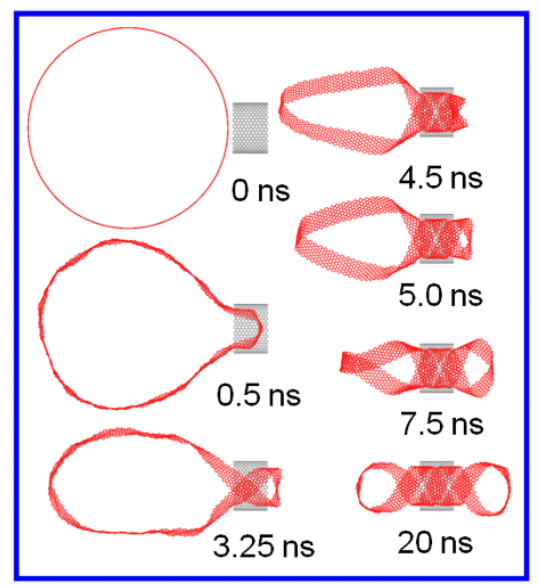

Figure 3 Representative snapshots of a CNR $(100,100)$ inserting into the SWCNT $(25,25)$ with the length of $24.60 \AA$ to form a helical configuration. The length of the CNR is $14.76 \AA$.

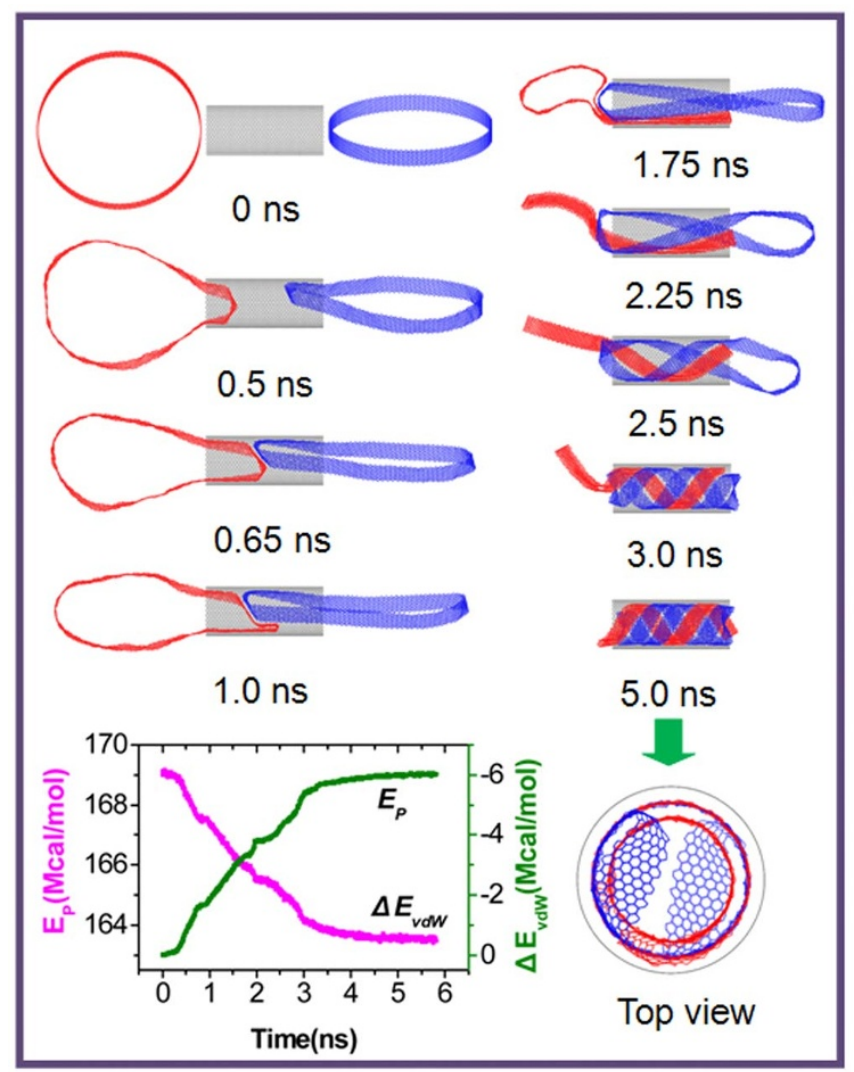

Figure $4 \mid$ Evolution snapshots of two CNRs $(100,100)$ inserted into the SWCNT $(30,30)$. The lengths of the CNRs are both $14.76 \AA$. The inset is the total potential energy $\left(\mathrm{E}_{\mathrm{P}}\right)$ and the $\mathrm{vdW}$ interaction energy $\left(\Delta \mathrm{E}_{\mathrm{vdW}}\right)$ between the CNR and SWCNT as a function of time.

SWCNT to form a helix. It is found that the chemical groups $-\mathrm{OH}$ have a certain influence on the interval between the neighboring segments. As the CNR $(100,100)$ without modification has 200 dangling $\sigma$-orbitals at one end, the possible number of the functional groups to modify the CNR is $0-400$. Then a series of simulations are performed on the CNRs $(100,100)$ modified with different number of $-\mathrm{OH}$, as shown in Figure 6(a). When one end of the CNR is modified with 50,100 and $133-\mathrm{OH}$, the CNR can form a helix in the SWCNT. In contrast, the CNR fully modified with $200-\mathrm{OH}$ at one end cannot form a helix, and the CNR modified with $400-\mathrm{OH}$ even cannot be trapped by the hollow interior of the SWCNT. In addition to the $-\mathrm{OH}$, we also chemically attach $-\mathrm{H}$ to the $\mathrm{CNR}(100,100)$ with the length of $14.76 \AA$ uniformly. In Figure 6(a), it is shown that when

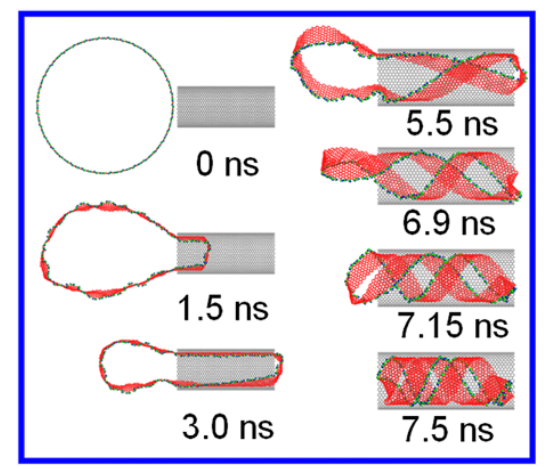

Figure $5 \mid$ Evolution snapshots of the $\mathrm{CNR}(100,100)$ modified with $-\mathrm{OH}$ entering with the SWCNT $(30,30)$. The length of the CNR is $14.76 \AA$ and the number of the $-\mathrm{OH}$ is 133 . 




Figure 6 | (a) Final configuration of CNR $(100,100)$ modified with functional groups encapsulated into the SWCNT $(30,30)$. The length of CNR is $14.76 \AA$; Total potential energy $\left(\mathrm{E}_{\mathrm{P}}\right)$ of the CNR-SWCNT systems as a function of time: (b) the CNR modified with $200-\mathrm{OH}$ and (c) the CNR modified with $133-\mathrm{OH}$.

the dangling $\sigma$-orbitals on the carbon atoms are all saturated by hydrogen atoms, the CNR can still be encapsulated into the SWCNT and form a helix, which is quite different from the final configuration of the CNR modified with - OH. In addition, the helixformation of the CNR without any dangling $\sigma$-orbitals also suggests that the Van der Waals interaction is the main driving force in forming a helical configuration, rather than the dangling $\sigma$-orbitals on carbon atoms at the end of $\mathrm{CNRs}^{30}$. We also study the stability of final configuration in different systems, in which the CNR is modified with a different number of -OH. As illustrated in Figure 6(b), we find that the whole potential of the $200-\mathrm{OH}$ system decreases to a certain value and maintains at this level for $7 \mathrm{~ns}$, which indicates that the configuration without helix is comparably stable. The potential in the system of CNR modified with $200-\mathrm{OH}$ is very different from that in the system of a helix configuration (Figure 6(c)). The potential energy in the helix-forming process has evident periods: two "rapid drop" periods, a "platform" period and the equilibrium state. It may be suspected that the modification of the CNR with different functional groups can influence the final configuration in the encapsulation process.

The above studies mainly focus on the insertion feature of the CNRs into the hollow SWCNTs. In this section, we will investigate the collapse and wrapping characteristic of the CNRs around the SWCNT. Figure 7(a) shows the typical snapshots of the CNR (80, 80) with the length of $14.76 \AA$ spontaneously wrapping on the SWCNT $(15,15)$. Initially, the CNR positioned in the center of the SWCNT, with the axis of the CNR aligned parallel to that of the SWCNT and the separation distance of about $5 \AA$ above the SWCNT. As shown in Figure 7(a), the CNR approaches the SWCNT rapidly due to the strong attractive force. During the approaching process, the CNR gradually stretches its cross-section from a circle to an oval, because the carbon atoms close to the SWCNT can endure stronger Van der Waals force than the upper one. After the CNR contacts with the SWCNT, the upper atoms continue approaching the SWCNT, inducing the oval cross-section to collapse to a " $\Delta$ " shape ${ }^{31}$, completely covering the surface of the

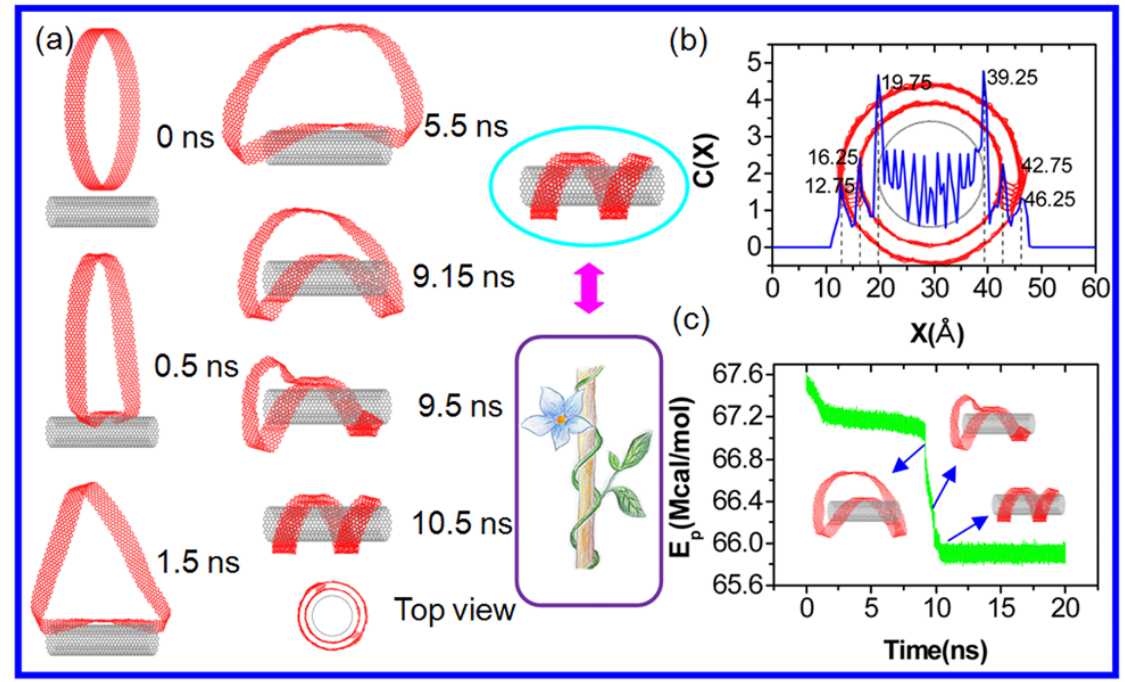

Figure $7 \mid$ (a) Representative snapshots of a CNR $(80,80)$ wrapping on the SWCNT $(15,15)$ to form a spiral configuration, which resembles the tendril climbing around a trunk (hand drawn sketches by Wei Chen). The length of the CNR is $14.76 \AA$ and the one of the SWCNT is $73.79 \AA$. (b) Concentration distribution profiles of the CNR and SWCNT in the system in the X-direction. (c) Total potential energy ( $\mathrm{E}_{\mathrm{P}}$ ) of the CNR-SWCNT systems as a function of time. 
Table 1 | The final configuration of CNRs with different diameters adhering on the SWCNT $(15,15)$. The lengths of the CNRs and the SWCNTs are $14.76 \AA, 73.79 \AA$ respectively

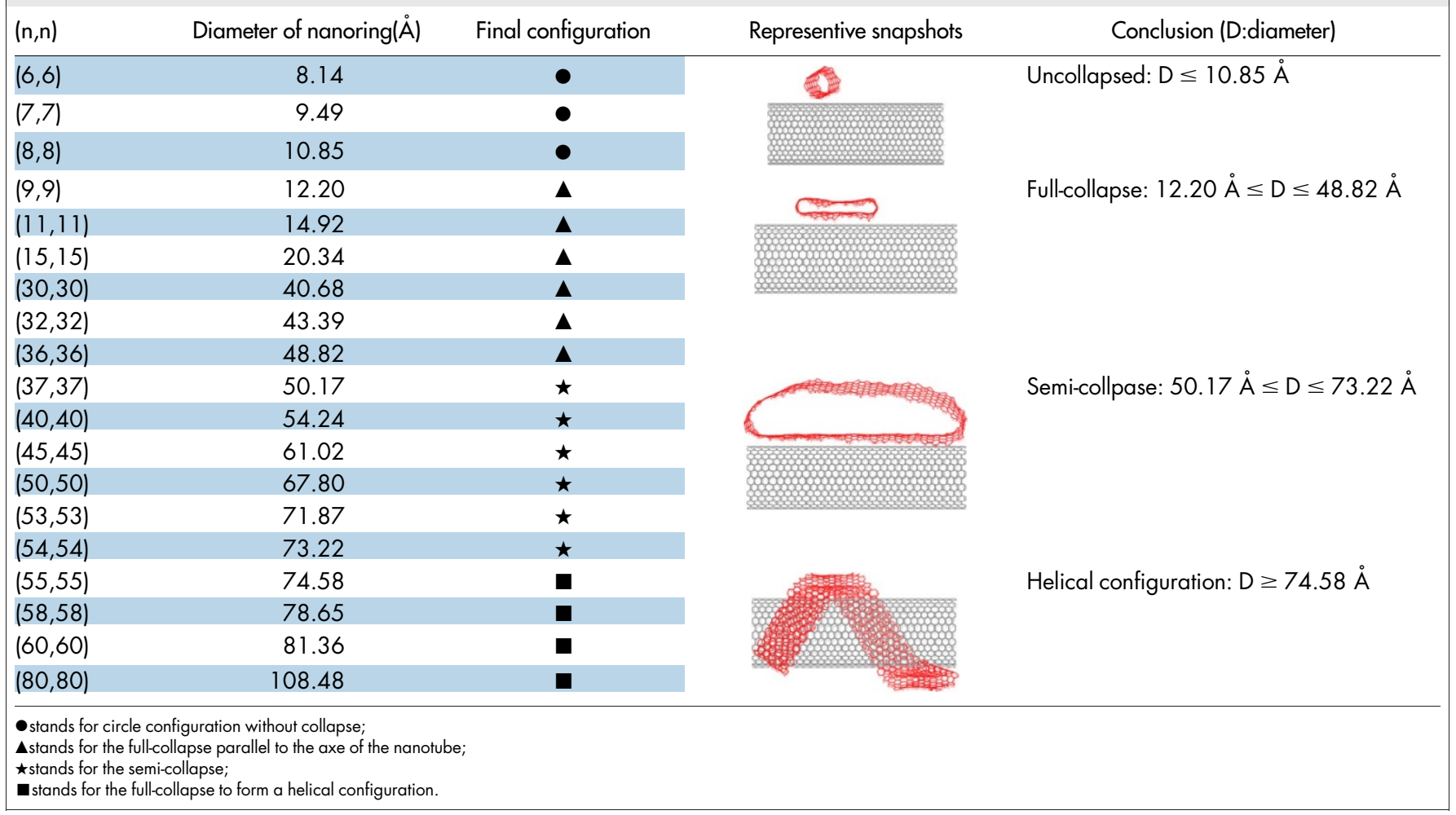

SWCNT. Then the axes of the CNR and SWCNT are staggered and the cross-section changes into a bow-shape. During this process, the Van der Waals interaction on the top semicircular tube is so weak that it makes the CNR collapse rather slowly. Eventually, at 9 ns, the collapse of the entire CNR is further accelerated due to the increase of the contact area $^{32}$. The sequential collapse of these rings forms a linked double graphitic nanoribbon, showing a double-walled helix wrapping around the SWCNT with a large helical pitch. Our results show that regardless of whether the axes of the CNR and SWCNT are perpendicular or parallel to each other in the initial models, the CNR can wrap around the SWCNT helically. The helix of the CNR adhering on the wall of the SWCNT resembles the tendril of a morning glory climbing around a tree trunk. This growth pattern helps the plant maximize the sunlight absorption in the minimum space and grow strong. It will be difficult to separate it with the trunk even in the strong wind.

We also study the concentration distribution profiles of the wrapping process in the X-direction and total potential energy $\mathrm{E}_{\mathrm{P}}$ of the CNR-SWCNT system as a function of time. As shown in Figure 7(b), the distance between two layers of the CNR outside the tube is also about $3.5 \AA$ A. The potential energy shown in Figure 7(c) has a decreasing tendency with the simulation time, and reaches the mimimum at equilibrium.

The final configurations of the CNRs with different diameters adhering on the SWCNTs are summarized in Table 1. We set the lengths of all SWCNTs $(15,15)$ as the same figure, i.e. $73.79 \AA$, and set the lengths of all CNRs to be $14.76 \AA$. As illustrated in Table 1, all CNRs with diameters larger than the threshold of $12.20 \AA$ are collapsed. However, CNRs with diameters between 50.17 and $73.22 \AA$ cannot fully collapse, and instead simply deform their cylindrical symmetry to bow-shapes. During this process, the Van der Waals energy cannot overcome the energy required for the fully mechanical deformation of the CNRs, leading to a mechanically bistable configuration $^{33}$. However, what we wish to know is how the CNRs spontaneously collapse to form ribbons and wrap on the SWCNT helically.
In order to explore the possible effects of the length of the SWCNTs on the final configuration, SWCNTs $(15,15)$ with different length and CNRs $(45,45)$ with length of $14.76 \AA$ are selected. As shown in Figure 8, it is clear that the CNRs with diameters of $61.02 \AA$ cannot collapse fully when the length of the SWCNTs reaches $63.95 \AA$ A. Due to the fact that we cannot determine all the CNRs with different diameters and SWCNTs with different lengths, Table 1 and Figure 8 suggest that if the lengths of the SWCNTs are smaller than the diameters of the CNRs, the perfect helix can be guaranteed. Figure 8(d) also illustrated that the SWCNTs with sufficient lengths can cause the CNRs to collapse as a linked double graphitic layer paralleled to the axe of the SWCNTs.

\section{Discussion}

In this study, a series of atomistic simulations have been performed to study the self-assembly of the CNR-SWCNT systems. The CNR can spontaneously insert into the inner cavity of the SWCNT to form a DNA-like double-helix. Furthermore, the CNR can also collapse to a double enclosed graphitic nanoribbon adhering on the outside wall

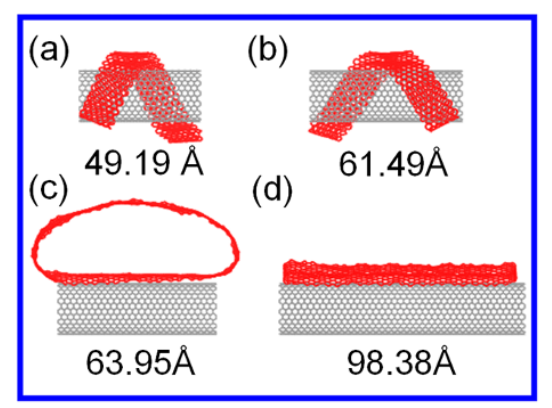

Figure $8 \mid$ Final configuration of CNRs $(45,45)$ with the diameter of $61.02 \AA$ adhering on the SWCNTs $(15,15)$ with different lengths. The length of the CNR is $14.76 \AA$. 
in a spiral form. In principle, the geometric structure transformation is determined by the competition between the van der Waals interaction energy and the bending strain energy of the $\mathrm{CNR}^{32}$. The competition between the Van der Waals energy, which provide an attractive force to collapse the CNR, and the elastic energy, which usually tends to keep the CNR in a circular form ${ }^{33}$, could lead to the circle configuration, fully collapsed configuration, or even mechanically bistable configuration of a CNR in which the two competitive energies are comparable. During the self-assembly process, it is the Van der Waals interaction between the CNR and the SWCNT, which helps the CNR overcome the energy barrier so that it may collapse $\mathrm{s}^{30,34}$, and drives the CNR to be trapped in the tube and undergo self-scrolling. Taking the insertion process as an example, as shown in Figure 9(a), the negative $\Delta \mathrm{E}_{\mathrm{vdW}}$, indicating an attractive force, suggests that the Van der Waals interaction between the CNR and SWCNT plays a dominant role in driving the continuous collapse of the CNR and forming spirals. Throughout the helix-forming course, the Van der Waals energy decreases significantly and finally reaches its minimum. The Van der Waals energy has partially transformed to the internal energy for mechanical deformation of CNR and partially converted into kinetic energy, thus sustaining the structure transition. As a result, the collapsed CNRs have the largest area to contact with the SWCNT, which reduces the systemic potential energy and enhances the stability of the CNR-SWCNT system.

We can explain well why the CNR can be trapped by the inner wall of the SWCNTs and collapse around the tube, but what surprises us is why the CNR enters or adheres on the SWCNT to form spirals. It can be speculated that two possible reasons are responsible for the spirals-forming process. The first reason is that the spiral pattern is the lowest energy state of the CNR-SWCNT system. To reveal it quantitatively, the total potential energy $E_{P}$ of the insertion process is shown in Figure 9(b). The decreased potential energy of the CNR-SWCNT system indicates that the helix-forming course is spontaneous, and that the system gradually reaches a more stable state. When the energy reaches its minimum, the entire system is in equilibrium and the perfect spirals are formed. It is worth noting that, during the inserting process, the curve has an evident "platform" in the period of 2 to $4 \mathrm{~ns}$, indicating that the contact area is virtually unchanged after the tube is fully filled between 2 and 4 ns. As far

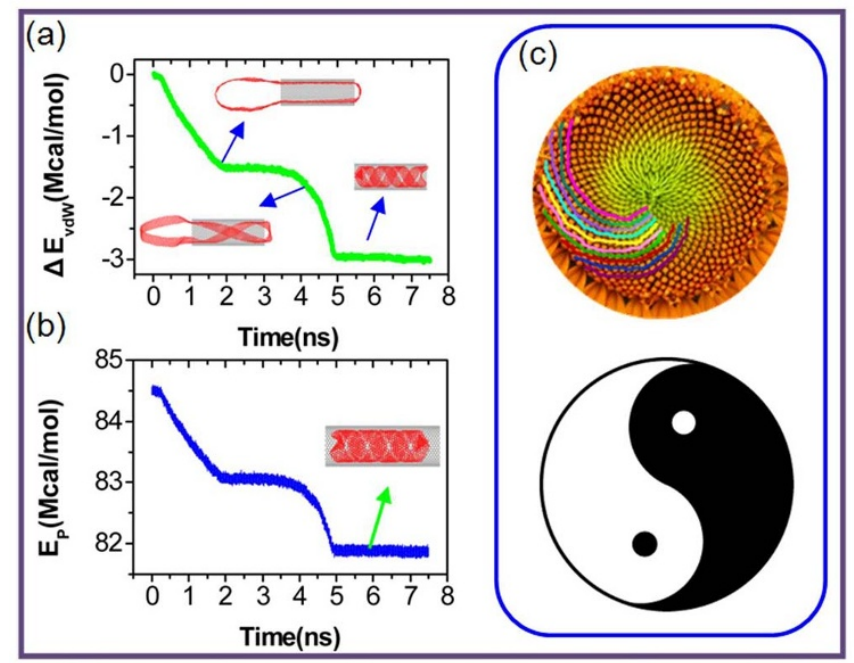

Figure $9 \mid$ (a) The vdW interaction energy differences $\left(\Delta \mathrm{E}_{\mathrm{vdW}}\right)$ between the CNR and SWCNT versus time in the insertion process. (b) Total potential energy $\left(E_{P}\right)$ of the CNR-SWCNT system as a function of time in the insertion process. (c) Spiral arrangement of florets in the sunflower (produced using Adobe Photoshop CS) and Chinese old Tai Chi totem (produced using Adobe Illustrator CS6). as the wrapping process is concerned, a "rapid drop", which occurred at about $9 \mathrm{~ns}$, is caused by the increase of the contact area, which corresponds to the accelerated collapse process. The second reason for this phenomenon is that the compact spirals of the CNR are the natural space savers which can achieve the maximum occupancy of the confined SWCNT. The spiral form of CNR encapsulated in the SWCNT is similar to the ordered, helical conformations of long molecular chains such as DNA and protein in the confined cell, and the wrapping process of the CNR resembles to the tendrils of the climbing plants. To better understand the helical configuration in the crowed environment, Snir and Kamien ${ }^{35}$ constructed a simulation system, in which a solid, impenetrable but flexible tube immersed in a solution of hard spheres. It is amazing that the flexible tube will take on a regular helix, which takes the least amount of energy and takes up the least space. Similarly, the spiral phenomenon is a relatively common but rather interesting phenomenon in the nature, such as spiral arrangement of florets in the sunflower (Figure 9(c)), the spiral nucleation of $\mathrm{SiC}$ crystal in a limited space, and the spiral arrangement of thunderstorms in the center of a tropical cyclone. What's more, Chinese old Tai Chi totem (Figure 9(c)) also emphasizes the philosophy of helical configuration, which implies that the universe derives from a series of helix. It is believed that helix is one of the essential features of materials and life even though its origin remains unclear.

To conclude, the CNR can be helically inserted into the SWCNT or wrap around the tube in a spiral form. It is suspected that the spiral configuration of the CNR takes the least amount of energy and takes up the least space. It is shown that the Van der Waals interaction between the CNR and the SWCNT plays a dominant role in driving the CNR to insert into the nanotube or wrap around the tube. The decrease of the potential energy in the CNR-SWCNT systems suggests that the helical insertion and wrapping processes are spontaneous. The sizes of the SWCNTs should exceed a certain threshold, to ensure the insertion of CNRs with certain lengths. Moreover, the diameters and lengths of the nanotubes have negligible effects on the encapsulation of the CNR to form a perfect helix. Two CNRs can also be encapsulated into the SWCNT spontaneously to form a helix. We also manifest that the modification of the CNR can influence the final configuration in the encapsulation process. Meanwhile, the results of our study also reveal that the sizes of the CNRs and SWCNTs should meet some required conditions to guarantee wrapping on the tube in a helical form.

The above-mentioned findings are of great importance toward the better understanding of CNT-SWCNT systems. The unique phenomenon of self-assembly in CNRs-SWCNTs systems has potential advantages in the application as nanocontainer for drug delivery, molecular transportation. This composite structure can be utilized to fabricate functional nanodevices, such as sensor, nanoelectronic components, integrated circuits, and optoelectronic devices.

\section{Methods}

In this paper, the force field of condensed-phased optimized molecular potentials for atomistic simulation studies (COMPASS) ${ }^{36}$ is used to model the atomic interaction. COMPASS is an ab initio force field which has been parameterized and validated using condensed-phase properties in addition to various ab initio calculations and experimental data, with a functional form that includes covalent terms as well as longrange, non-bond (Van der Waals $(\mathrm{VdW})$ ) interactions and electrostatic forces. The aims of the force field are to achieve high accuracy in predicting the properties of very complex mixtures $^{37}$ and it has been widely used due to its potential to obtain reasonable results in terms of the mechanical properties of $\mathrm{CNTs}^{38,39}$. The van der Waals energy is described by LJ-96 function ${ }^{37}$ in COMPASS forcefield, the functional forms of which is listed as: $E=D_{0}\left[2\left(\frac{R_{0}}{R}\right)^{9}-3\left(\frac{R_{0}}{R}\right)^{6}\right]$, where $D_{0}=0.064 \mathrm{Kal} / \mathrm{mol}$, and $R_{0}=$ $4.01 \AA^{40,41}$. A constant volume and constant temperature dynamics (NVT) ensemble is used in our molecular dynamics simulations with temperature of $300 \mathrm{~K}$. The Andersen method ${ }^{42}$ in the thermostat is employed to control the temperature and generate the correct statistical ensemble. For temperature control, the thermodynamic temperature is kept constant by allowing the simulated system to exchange energy using a "heat bath". The Verlet algorithm is adopted to integrate the equations of motion for the entire system. The time step is chosen to be $1.0 \mathrm{fs}$, and the data are 
collected every 5 ps to record the full-precision trajectory for further analysis. The SWCNTs are fixed as rigid tube structures. The CNRs are super-short CNTs with a length down to the molecular scale.

1. Zhao, X. C. \& Johnson, J. K. Simulation of adsorption of DNA on carbon nanotubes. J. Am. Chem. Soc. 129, 10438-10445 (2007)

2. Tsang, S. C., Chen, Y. K., Harris, P. J. F. \& Green, M. L. H. A simple chemical method of opening and filling carbon nanotubes. Nature 372, 159-162 (1994).

3. Ajayan, P. M., Stephan, O., Redlich, P. \& Colliex, C. Carbon nanotubes as removable templates for metal oxide nanocomposites and nanostructure. Nature 375, 564-567 (1995).

4. Li, H. et al. Carbon nanotube seeded silicon crystal growth. Appl. Phys. Lett. 95, 063106 (2009)

5. Pederson, M. R. \& Broughton, J. Q. Nanocapillarity in fullerene tubules. Phys. Rev. Lett. 69, 2689-2692 (1992).

6. Ajayan, P. M., Ebbesen, T. W., Ichihashi, T., Iijima, S. \& Tanigaki, K. Opening carbon nanotubes with oxygen and implications for filling. Nature 362, 522-525 (1993).

7. Borawiak-Palen, E. et al. Iron filled single-wall carbon nanotubes-a novel ferromagnetic medium. Chem. Phys. Lett. 421, 129-133 (2006).

8. Maniwa, Y. et al. Water-filled single-wall carbon nanotubes as molecular nanovalves. Nat. Mater. 6, 135-141 (2007).

9. Zou, J., Ji, B. H., Feng, X. Q. \& Gao, H. J. Molecular-dynamic studies of carbonwater-carbon composite nanotubes. Small 2, 1348-1355 (2006).

10. Smith, B. W., Monthioux, M. \& Luzzi, D. E. Encapsulated C60 in carbon nanotubes. Nature 396, 323-324 (1998).

11. Troche, K. S. et al. Prediction of ordered phases of encapsulated C60, C70, and C78 inside carbon nanotubes. Nano Lett. 5, 349-355 (2005).

12. Jiang, Y. Y., Li, H. \& Li, Y. F. Helical encapsulation of graphene nanoribbon into carbon nanotube. ACS Nano 5, 2126-2133 (2011).

13. Chuvilin, A. et al. Self-assembly of a sulphur-terminated graphene nanoribbon within a single-walled carbon nanotube. Nature 10, 687-692 (2011)

14. Zheng, M., Anand, J. \& Michael, S. S. Structure-based carbon nanotube sorting by sequence-dependent DNA assembly. Science 302, 1545-1548 (2003).

15. Brittany, G., Brenda, S., Donald, S. B., Robert, M. S. \& Jennifer, N. C. Sequenceindependent helical wrapping of single-walled carbon nanotubes by long genomic DNA. Nano Lett. 6, 159-164 (2006).

16. Baskaran, D., Mays, J. W. \& Bratcher, M. S. Noncovalent and nonspecific molecular interactions of polymers with multiwalled carbon nanotube. Chem. Mater. 17, 3389-3397 (2005).

17. Nish, A., Hwang, J. Y., Doig, J. \& Nicholas, R. J. Highly selective dispersion of single-walled carbon nanotubes using aromatic polymers. Nat. Nanotechnol. 2, 640-646 (2007).

18. Chen, R. J., Zhang, Y., Wang, D. \& Dai, H. Noncovalent sidewall functionalization of single-walled carbon nanotubes for protein immobilization. J. Am. Chem. Soc. 123, 3838-3839 (2001)

19. Choi, W. Y., Kang, J. W. \& Hwang, H. J. Structure of ultrathin copper nonawires encapsulated in carbon nanotubes. Phys. Rev. B 68, 193405 (2003)

20. Planeix, J. M. et al. Application of carbon nanotubes as supports in heterogeneous catalysis. J. Am. Chem. Soc. 116, 7935-7936 (1994).

21. Singh, R. et al. Binding and condensation of plasmid DNA onto functionalized carbon nanotubes: toward the construction of nanotube-based gene delivery vectors. J. Am. Chem. Soc. 127, 4388-4396 (2005).

22. Sun, F. W. \& Li, H. Torsional strain energy evolution of carbon nanotubes and their stability with encapsulated helical copper nanowires. Carbon 49, 1408-1451 (2011).

23. Sun, J. et al. Carbon nanorings and their enhanced lithium storage properties. $A d v$. Mater. 25, 1125-1130 (2013)

24. Elliott, J. A. et al. Collapse of single-wall carbon nanotubes is diameter dependent. Phy. Rev. Lett. 92, 095501 (2004)

25. Gao, G. H., Cagin, T. \& Goddard, W. A. Energetics, structure, mechanical and vibrational properties of single-walled carbon nanotubes. Nanotechnology 9 , 184-191 (1998).

26. Lu, J. Q. et al. Metal-to-semiconductor transition in squashed armchair carbon nanotubes. Phys. Rev. Lett. 90, 156601 (2003).
27. Chopra, N. G. et al. Fully collapsed carbon nanotubes. Nature 377, 135-138 (1995).

28. Sun, X. M. et al. Optical properties of ultrashort semiconducting single-walled carbon nanotube capsules down to sub-10 nm. J. Am. Chem. Soc. 130, 6551-6555 (2008).

29. Ruoff, R. S., Tersoff, J., Lorents, D. C., Subramoney, S. \& Chan, B. Radial deformation of carbon nanotubes by van der Waals forces. Nature 364, 514-516 (1993)

30. Li, Y. F., Sun, F. W. \& Li, H. Helical wrapping and insertion of graphene nanoribbon to single-walled carbon nanotube. J. Phys. Chem. C 115, 18459-18467 (2011).

31. Yan, K. Y. et al. Radial collapse of single-walled carbon nanotubes induced by the $\mathrm{Cu}_{2} \mathrm{O}$ surface. J. Phys. Chem. C 113, 3120-3126 (2009).

32. Yan, K. Y. et al. The core/shell composite nanowires produced by self-scrolling carbon nanotubes onto copper nanowires. ACS NANO 3, 2235-2240 (2009).

33. Lu, W. B. \& Chou, T. W. Radial deformation and its related energy variations of single-walled carbon nanotubes. Phys. Rev. B 83, 134113 (2011).

34. Zhang, S. L., Khare, R. \& Belytschko, T. Transition states and minimum energy pathways for the collapse of carbon nanotubes. Phy. Rev. B 73, 075423 (2006).

35. Snir, Y. \& Kamien, R. D. Entropically driven helix formation. Science 307, 1067 (2005).

36. Sun, H. COMPASS: an ab initio force-field optimized for condensed-phase applications overview with details on alkane and benzene compounds. J. Phys. Chem. B 102, 7338-7364 (1998).

37. Bunte, S. W. \& Sun, H. Molecular modeling of energetic materials: the parameterization and validation of nitrate esters in the compass force field. J. Phys. Chem. B 104, 2477-2489 (2000)

38. Wang, Q., Duan, W. H., Liew, K. M. \& He, X. Q. Inelastic buckling of carbon nanotubes. Appl. Phys. Lett. 90, 033110-1-3 (2007).

39. Wang, Q. Atomic transportation via carbon nanotubes. Nano. Lett. 9, 245-249 (2009).

40. Sun, H., Mumby, S. J., Maple, J. R. \& Hagler, A. T. An ab initio CFF93 all-atom force field for polycarbonates. J. Am. Chem. Soc. 116, 2918-2981 (1994).

41. Rigby, D., Sun, H. \& Eichinger, B. E. Computer simulations of poly(ethylene oxide): force field, PVT diagram and cyclization behaviour. Polym. Int. 44, 311-330 (1998)

42. Andersen, H. C. MDs simulations at constant pressure and/or temperature. J. Chem. Phys. 72, 2384-2393 (1980).

\section{Acknowledgments}

We would like to acknowledge the support from the National Natural Science Foundation of China (Grant No. 51271100) and the National Basic Research Program of China (Grant No.2012CB825702). This work is also supported by the Special Funding in the Project of the Taishan Scholar Construction Engineering.

\section{Author contributions}

W.C. performed all simulations and prepared the manuscript. W.C. and H.L. designed the research, discussed the results, drew conclusions and edited the manuscript

\section{Additional information}

Supplementary information accompanies this paper at http://www.nature.com/ Scientificreports

Competing financial interests: The authors declare no competing financial interests.

How to cite this article: Chen, W. \& Li, H. How Does Carbon Nanoring Deform to Spiral Induced by Carbon Nanotube? Sci. Rep. 4, 3865; DOI:10.1038/srep03865 (2014).

cc) (i) $\Theta$ This work is licensed under a Creative Commons AttributionNonCommercial-NoDerivs 3.0 Unported license. To view a copy of this license, visit http://creativecommons.org/licenses/by-nc-nd/3.0 